\title{
Congenital tuberculosis: presentation of a rare case
}

\author{
Asst. Prof. Velat Şen, M.D. ${ }^{a}$, Asst. Prof. Hadice Selimoğlu Şen, M.D. ${ }^{b}$, Asst. Prof. Fesih Aktar, M.D. ${ }^{c}$, \\ Asst. Prof. Ünal Uluca, M.D. ${ }^{c}$, Asst. Prof. Müsemma Karabel, M.D. ${ }^{a}$ and Prof. Mehmet Fuat Gürkan, M.D. ${ }^{a}$
}

\begin{abstract}
Congenital tuberculosis is a rare disease with a high mortality rate. Congenital tuberculosis is considered the result of motherto-child transmission from the placenta to the fetus, through the ingestion of the amniotic fluid, or via transplacental transmission through the umbilical vein. Given the non-specific clinical signs of tuberculosis, it is usually difficult to diagnose it. The case of a 48-day-old male infant hospitalized due to weight loss, fever, cough, hemoptysis, and respiratory distress for the past 20 days, is presented. In this period, he had received broad spectrum antibiotics but with no improvement. A chest $\mathrm{x}$-ray showed the presence of consolidation and a cavitary lesion in the upper and middle left lung fields. Mycobacterium tuberculosis was detected by polymerase chain reaction in a bronchoalveolar lavage specimen. Congenital tuberculosis was diagnosed based on this finding; hence, a tuberculostatic regimen was started accordingly. The patient died 13 days after treatment initiation.

Congenital tuberculosis should be considered in infants with weight loss, fever, cough, hemoptysis and respiratory distress. Key words: child, congenital tuberculosis, mortality.
\end{abstract}

http:/ / dx.doi.org/10.5546/aap.2015.eng.e101

\section{INTRODUCTION}

Intrauterine infection with tuberculosis bacillus is known as congenital tuberculosis. The World Health Organization reported that, in 2010, tuberculosis led to the death of half a million women and, at least, 64000 children. ${ }^{1}$ There were also some 10 million children orphaned by tuberculosis. ${ }^{1}$

According to the 2012 report on the situation of tuberculosis in Turkey, ${ }^{2}$ overall tuberculosis mortality was 3.1\% (525 deaths out of 17148 cases). Congenital tuberculosis has been frequently reported in regions with a high

a. Department of Pediatric Pulmonology.

b. Department of Pulmonology.

c. Department of Pediatrics.

Dicle University Medical School, Diyarbakir, Turquía.

E-mail Address:

Asst. Prof. Velat Şen, M.D.: drvelatsen@hotmail.com,

velat.sen@dicle.edu.tr

Conflict of Interest: None.

Received: 7-16-2014

Accepted: 9-22-2014 tuberculosis prevalence among adults. The high rate of tuberculosis among women of childbearing potential in developing countries is also related to a higher risk of congenital tuberculosis. The incidence of congenital tuberculosis is low; however, its significance lays on a mortality rate of up to $50 \%{ }^{3,4}$ In this article, a case of congenital tuberculosis accompanied by a paraspinal abscess is presented because of its infrequency and interesting radiographic findings.

\section{CLINICAL CASE}

A 48-day-old male infant was admitted to the hospital with the following symptoms: weight loss, fever, cough, hemoptysis and respiratory distress. Symptoms started when he was 28 days old. The infant was hospitalized due to suspected bacterial infection and was treated with broad spectrum antibiotics at different health care facilities over a 20-day period. In spite of the longterm antibiotic use, the infant's condition did not improve and was referred to a tertiary-care university hospital.

Based on the patient's history, it was established that he was a healthy term infant with low birth weight (2600 g) who had received no vaccines so far.

The initial postnatal period was normal, but at 28 days of life, he presented with progressive dyspnea, fever, anorexia and weight loss.

Upon admission to our hospital, the patient had a temperature of $38.6^{\circ} \mathrm{C}$, his heart rate was 164 beats per minute and his respiratory rate was 64 breaths per minute. He had severe tachypnea, dyspnea and subcostal and intercostal retractions. Bilateral rhonchi and rales were found upon pulmonary auscultation. Liver and spleen enlargement were not detected. A chest $x$-ray showed condensation areas with a cavitary lesion in the lower and middle left lung fields (Figure 1). Blood count results were as follows: leukocytes $23 \times 110^{9}$ cells/ $\mathrm{mm}^{3}$ (neutrophils: $36.8 \%$, lymphocytes: $48.2 \%$, monocytes: $11.5 \%$ ), hemoglobin $7.9 \mathrm{~g} / \mathrm{dL}$, and platelets $60 \times 110^{9}$ cells $/ \mathrm{mm}^{3}$. C-reactive protein and erythrocyte sedimentation rate values were $7.7 \mathrm{mg} / \mathrm{dL}$ and $41 \mathrm{~mm} / \mathrm{h}$, respectively. Serum electrolytes, and liver and kidney function tests were normal. Blood and urine cultures were 
negative for Mycobacterium tuberculosis (MT). A chest computed tomography showed condensation areas with an air bronchogram in the posterior region of the upper left lobe and a cavitation of $2 \mathrm{~cm}$ in diameter in the same area (Figure 2. A, B). Ground-glass opacity was also observed in the posterior region of the upper right lobe. Multiple images of the septum showed peripheral thickening of $2 \mathrm{~cm}$ on the right side and of $1.5 \mathrm{~cm}$ on the left side suggesting the presence of a paraspinal abscess in the left and right paravertebral areas of the last thoracic vertebra (Figure 2. C-F). The abdominal ultrasound was normal. Total Ig G, A and M levels were normal, so immunodeficiency was ruled out.

Tuberculosis infection was suspected given the lack of response to antibiotic therapy after five days. Therefore, gastric aspirate specimens were collected to study the presence of acidfast bacilli (AFB). Specimens were negative for AFB using Ziehl-Neelsen staining. No endobronchial lesions were observed in the bronchoscopy. M. tuberculosis was detected by polymerase chain reaction in the specimens collected by bronchoalveolar lavage nine days following hospitalization. Treatment was started with isoniazid, rifampicin, and pyrazinamide. Although a mild improvement was observed seven days after treatment initiation with tuberculostatic agents, the patient died on day 13 following an episode of massive hemoptysis. The culture of the bronchoalveolar lavage specimen was positive for $M$. tuberculosis 40 days after its collection.

FIGURE 1 . Chest $x$-ray showing a condensation area and a cavitary lesion in the left upper and middle regions

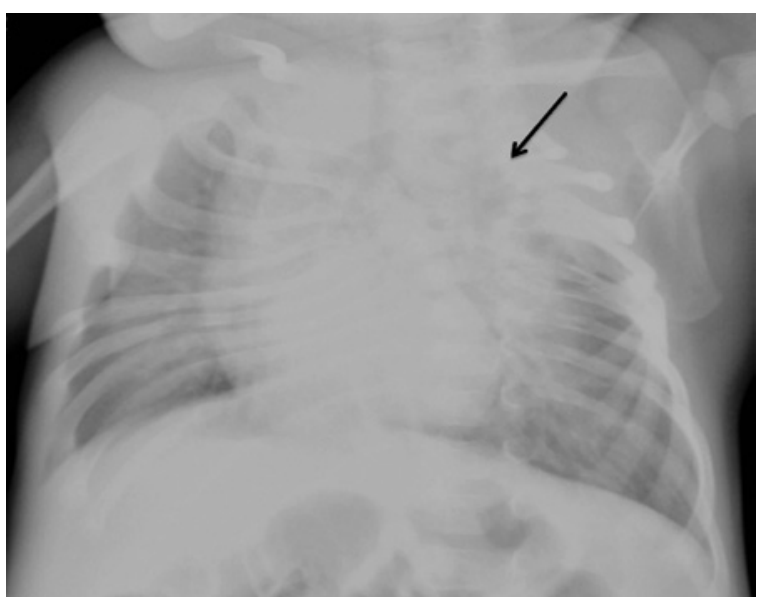

\section{Maternal history}

The patient's mother was 31 years old and had no symptoms throughout the pregnancy and the postpartum period. Once suspected, pulmonary tuberculosis was confirmed and she started receiving treatment. She referred frequent contact with an aunt who had a history of tuberculosis.

\section{COMMENT}

There are two main infection routes for congenital tuberculosis. Firstly, it can be transmitted from the mother to the fetus via transplacental transmission through the umbilical vein, thus forming a primary MT complex in the liver of the child with secondary hematogenous spread. ${ }^{5}$ Secondly, amniotic fluid aspiration or intake leads to the formation of a primary MT complex in the gastrointestinal tract or the lungs. ${ }^{5}$ The most commonly involved sites are the liver and the lungs. Signs and symptoms appear after three weeks of life, with a median age of 28 days. ${ }^{6}$

Congenital tuberculosis should be considered when a child's signs are indicative of this disease and his/her mother has a history of $M$. tuberculosis infection or signs suggestive of infection. However, up to $60-70 \%$ of mothers have no symptoms and newborn infants usually have non-specific signs and symptoms..$^{6-8} \mathrm{~A}$ recent study by Peng, et al. found that 162 mothers had active tuberculosis during pregnancy or the postpartum period. Of them, 121 had no history of tuberculosis before getting pregnant and were diagnosed during pregnancy. ${ }^{9}$ The mother in this case had no symptoms and was diagnosed as a result of looking for the source of tuberculosis in her child. Criteria to distinguish between congenital and postpartum tuberculosis were first clearly established by Wallgren and then reviewed by Cantwell, et al. in 1994,6,10 (Table 1).

Clinical signs in infected newborn infants are usually non-specific. Pneumonia, sepsis and purulent meningitis caused by congenital tuberculosis cannot be distinguished from those caused by other germs, so it is usually under-diagnosed. ${ }^{9}$ The presence of liver, spleen and lymph node enlargement may guide the diagnosis, but this is not often the case. ${ }^{11}$

In our case, the normal abdominal ultrasound suggests that the primary source of infection was the lung.

Congenital tuberculosis should be suspected when antibiotics do not work and tests for other congenital infections are negative, especially if the mother has a history of tuberculosis or has been 
FiGURE 2. Chest computed tomography showing condensation and cavitation areas in the posterior region of the upper left lobe (arrows shown in A and B). Paraspinal abscess in the paravertebral area at the level of lower thoracic vertebrae (arrows shown in $C-F)$
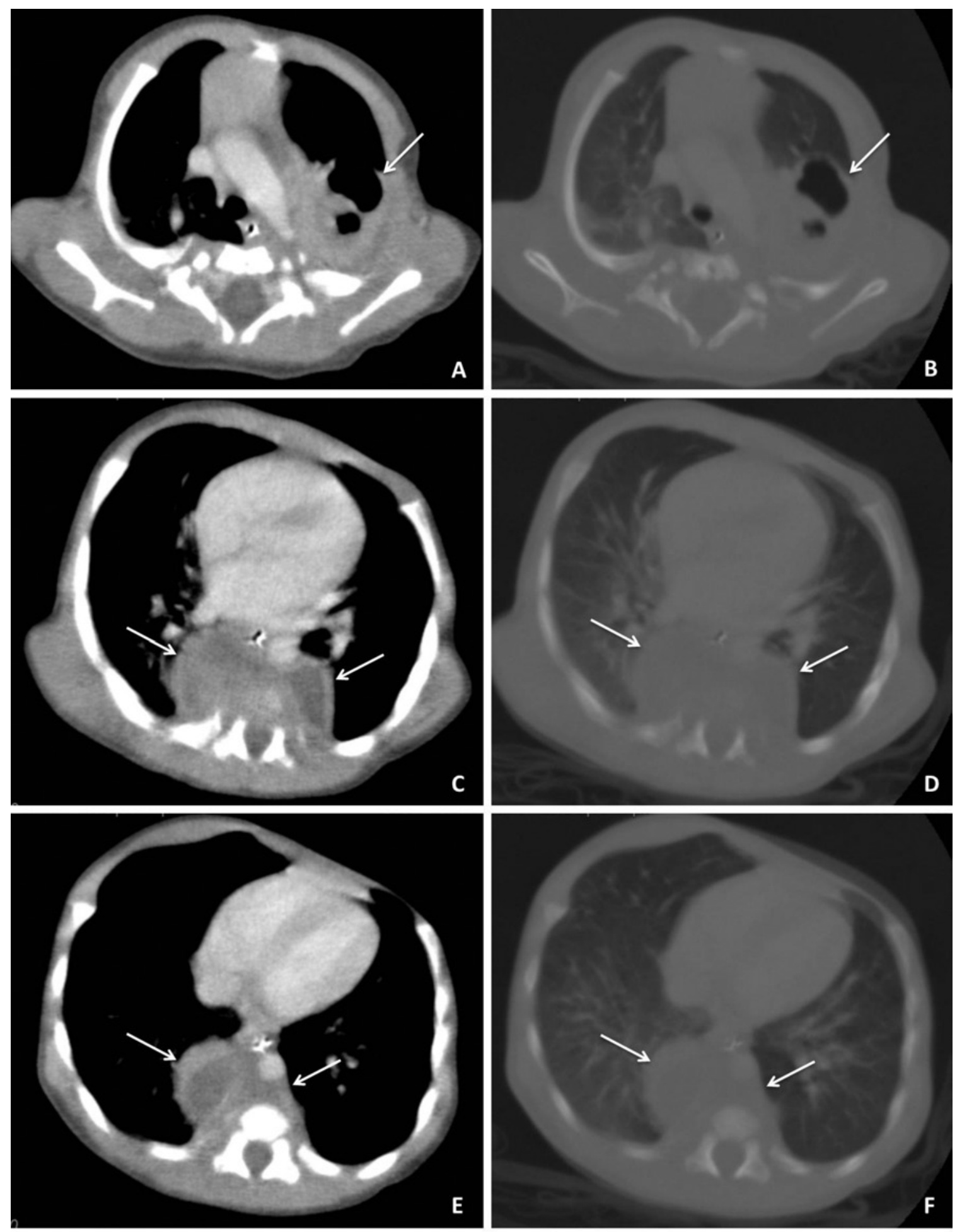
recently diagnosed. In relation to tuberculosis diagnosis, tuberculin tests are positive in less than $15 \%$ of tuberculosis cases and may take from one to three months to turn positive. ${ }^{6,12}$ In our case, the test was negative. Diagnosis depends on clinical suspicion and on demonstrating the presence of AFB in tissues or fluids. In our case, tuberculosis was first determined by polimerase chain reaction and by bronchoalveolar lavage culture. No tuberculosis bacilli were detected in this patient's urine and blood samples, possibly because there was no spread during the postnatal period.

Most infants have a normal chest x-ray. Radiographic findings may vary from interstitial pneumonia, consolidation and cavitation images to a miliary pattern. In general, $x$-rays are not helpful for making the diagnosis. ${ }^{11}$ In our patient, the x-ray showed multiple consolidation areas and cavitary lesions. This patient also had non-specific signs and symptoms, which resembled those of bacterial sepsis. Diagnosis was exclusively based on a high suspicion following the failure of broad spectrum antibiotics and after immunodeficiency was ruled out.

Singh, et al. reported clinical and laboratory findings that may suggest congenital tuberculosis, which include newborn infants born in tuberculosis endemic areas with pneumonia poorly responsive to antibiotics, maternal tuberculosis, and infants with non-specific symptoms. ${ }^{13}$ Our patient met all three criteria.

\section{CONCLUSION}

This case exemplifies the possibility of congenital tuberculosis in children with infections who do not respond favorably to antibiotic therapy. A detailed clinical history is particularly helpful in those regions with a high prevalence of tuberculosis. Even when there is no maternal history, this diagnosis should not be excluded in infants. Given its wide variability, congenital tuberculosis may be under-diagnosed. Congenital and perinatal tuberculosis should be considered, especially in developing countries where there is a high prevalence of this disease.

\section{Acknowledgments}

We would like to thank Abdurrahim Dusak, M.D., from the Dicle University Medical School Department of Radiology, for providing radiographic images.

\section{REFERENCES}

1. World Health Organization. Global tuberculosis report 2012. Geneva: World Health Organization; 2012. Available at:http:/ / www.who.int/tb/publications/global_report/ gtbr12_main.pdf. [Accessed on: September 24, 2014].

2. Ministry of Health of Turkey. 2012 Report on situation of tuberculosis in Turkey: Analysis of Treatment Results:89-102. Ankara: Ministry of Health of Turkey; 2013. Available at: Türkiye'de Verem Savaş Raporları. [Accessed on: September 24, 2014].

3. Crockett M, King SM, Kitai I, Jamieson F, et al. Nosocomial transmission of congenital tuberculosis in a neonatal intensive care unit. Clin Infect Dis 2004;39(11):1719-23.

4. Starke JR. Tuberculosis. An old disease but a new threat to the mother, fetus, and neonate. Clin Perinatol 1997;24(1):10727.

5. HagemenJ,ShulmanS,Schreiber M,LuckS, etal.Congenital tuberculosis: critical reappraisal of clinical findings and diagnostic procedures. Pediatrics 1980;66(6):980-4.

6. Cantwell MF, Sehab ZM, Costello AM, Sands L, et al. Brief report: congenital tuberculosis. $N$ Engl J Med 1994;330(15):1051-4.

TABLE 1. Diagnostic criteria for congenital tuberculosis

\section{Wallgren's criteria}

a. Primary lesion in the liver or satellite lymph nodes corresponding to the portal system.

b. The lung is the primary source of infection (aspiration) and confirms that the infection did not occur in the postnatal period.

\section{Cantwell's criteria}

Main criterion: Tuberculous lesions should be confirmed in the infant.

Secondary criteria:

1. Lesions developed in the first week of life.

2. Primary hepatic complex or caseating hepatic granulomas.

3. Tuberculosis infection in the placenta or maternal genital tract.

4. Ruling out the possibility of postnatal transmission by investigating who came into contact with the infant, including hospital staff, and in order to comply with existing recommendations that establish providing treatment o infants exposed to tuberculosis. 
7. Hassan G, Qureshi W, Kadri SM. Congenital tuberculosis. JK Sci J Med Educ Res 2006;8:193-4.

8. Adhikari M, Pillay T, Pillay DG. Tuberculosis in the newborn: an emerging disease. Pediatr Infect Dis J 1997;16(12):1108-12.

9. Peng W, Yang J, Liu E. Analysis of 170 cases of congenital TB reported in the literature between 1946 and 2009. Pediatr Pulmonol 2011;46(12):1215-24.
10. Wallgren AJ. Tratado de tuberculosis infantil. Buenos Aires: El Ateneo; 1940:27-9.

11. Pillay T, Adhikari M. Congenital tuberculosis in a neonatal intensive care. Clin Infect Dis 1999;29(2):467-8.

12. Ormerod P. Tuberculosis in pregnancy and the puerperium. Thorax 2001;56(6):494-9.

13.Singh M, Kothur K, Dayal D, Kusuma S. Perinatal tuberculosis: a case series. J Trop Pediatr 2007;53(2):135-8. 